\title{
Wine, wineries \& internet: How new media are used by producers of Langhe and Roero
}

\author{
Andrea Viberti, Stefano Massaglia ${ }^{\mathrm{a}}$, and Danielle Borra \\ Università di Torino, DISAFA, Largo Paolo Braccini, 2, 10095 Grugliasco (TO), Italia
}

\begin{abstract}
The evolution of the society and of its habits, the advent of the technology and of new forms of communication and social networks is gradually changing the way that communication happens between the consumer and the company as more importance has been given to the network and to the many facets that it can offer. Social networks now represent an important resource for an easy and immediate connection with a large number of consumers easy. Another important feature is the ability to get a direct contact with a target characterized by an interest in the product, so that fewer resources are dispersed. Thanks to this work, it was possible to check the on-line presence of a large number of companies of Langa and Roero areas, and to evaluate the usage of such channels and the perceived importance about the contacts coming from the network. Moreover, it was possible to realize a first comparison with other areas like those of Cuneo.
\end{abstract}

\section{Introduction}

The evolution of the society and of its habits, the advent of the technology, of new forms of communication and of social is gradually changing the way that communication happens between the consumer and the company as more importance has been given to the network and to the many facets that it can offer. Internet sites are increasingly representing a good source of supply in the process of acquiring new customers and promoting the company, products and activities related to it. In addition, social networks have gradually gained importance, representing today a valuable resource to easily reach a large number of consumers. Another important feature, is given by the possibility of obtaining a direct contact with a target characterized by an interest in the product, so that fewer resources are dispersed. Blogs, video reviews and online shops are an additional form of innovation in the marketing process and in the wine trading which is undergoing a progressive transformation that is unavoidable for the companies. The Food Science Dept. of Turin University, studied the on-line presence of the wineries in the province of Cuneo in order to evaluate the structure of the different sites, the use of social networks and other advanced solutions employed in the presentation of the products and the companies as well as the response-time of the requests received through such channels. The hills of the Langhe, represent one of the most important Italian wine-growing areas: names such as Barolo and Barbaresco carry a very own history and a century-old tradition characterized by an exceptional level of quality that evident in more varieties of other wine products, such as Barbera d 'Alba, Nebbiolo,

$\overline{{ }^{a} \text { Corresponding author: stefano.massaglia@unito.it }}$
Verduno Pelaverga. To the scenery may be added the Arneis produced in the Roero hills and Monferrato areas, which have become famous for Barbera, the Asti and Moscato d'Asti.

Due to the uniqueness of the area, the production scenario and the strong innovative capacity of companies in June 2014, these areas have been recognized as a World Heritage Site by UNESCO.

\section{Material and methods}

After a brief research of similar activities in other world areas, an operating model has been identified to be taken in order to get results that can be compared with similar publications around the globe. The American Journal of Wine Research has published an interesting study [1] concerning marketing activities carried out through the Internet from which models operated to conduct the research were derived. In order to acquire a list of companies accessible to the public, three of the most important wine guides available on the market on the international scene have been chosen, "Slow Wine" [2] "Italian Wines" [3] "The Wines of Italy" [4]. By drafting a spreadsheet a list of 224 companies domiciled or operating branches in the province of Cuneo was created. With these data, it was possible to take into account, in a simple and accessible way, most of the companies operating in the area of the Langhe and Roero. Within the list, the presence of a corporate website, of an email, and of other general information useful to identify the company was added. At this stage, the list of parameters suitable to check every website, has been created. For companies where it was not indicated, in any of the books, a website, a search was 
carried out in the network in order to verify its presence. The main parameters recorded were as follows:

- Languages availability,

- Information availability,

- Presence of a online shop,

- Presence of blog or presence of Videoreviews,

- Social Networks links,

- Structure and type of the website;

- Chance to visit the winery;

- Opportunity for overnight stays;

The thorough examination of each website allowed us to draft a classification based on their functionality and content. To complete the study, an e-mail to contact the same for all companies was created: we asked information about the presence of the products in retail shops in the city of Rome, the opportunity to visit the winery and its costs and the possibility of purchasing products by email orders. Thanks to these emails it was possible to check the response-time and investigate the issues outlined above.

\section{Data analysis}

The companies identified, are all characterized by a high level of appreciation at an international level; it is then fundamental to give a strong attention to the presence on the Internet. A total of 6 companies have not developed a website yet; 12 websites at the time of writing were not accessible. A total of 206 websites were accessible and viewable. All companies have at least one email address. The international wine market suggests the presence of information, as well as in Italian, also in the other languages in order to facilitate access even to foreign users. The most widespread international language is definitely English used in 185 websites (89.8\%). Followed by German with 54 sites $(26.2 \%)$, by French with 17 sites $(8.2 \%)$, by Chinese with 5 sites $(2.4 \%)$, and by Spanish with 3 sites $(1.5 \%)$. The websites that have two languages usually allow you to choose the desired language with a special page before accessing the homepage. Those with multiple languages generally are loaded into the main one, allowing then to be able to change the display in the desired language. In both cases there are parallel versions with similar structure where only changes the text. The structure of the website varies depending on the companies and the information they want to give this way. The sites are very basic, kind a "old-fashioned" and based mainly on visual effects designed to show the views and the scenery of the company, others are more focused on the description of the company and its products instead. However, we can identify among all, fifty cases of companies that use an organizational model and structure made by a local company specialized in website construction and communication. In these sites only in the graphic and textual information differ while the structure is similar to all the others.

The content of the websites appears to be fairly standardized. In 195 websites, we have found a description focused on the history and evolution of the company, as well as the vision and the goals they want to achieve. In 198 websites we found instead the presence of products showcases, often combined with the ability to view and download the data sheet of the product. We can therefore say that in all the working websites there is a presentation of the products but not always of the company.

Another important point concerns the existence of a web section indicating the presence of retail shops, wine bars and restaurants where you can find the products. Even in this case there are very few companies that provide this information (17). One of the reasons is certainly linked to the difficulty of managing a database with all the stores, wine shops and restaurants which very often are not even direct customers of the company. Another point assessed during the visit of every web site, is the ability to purchase wine directly through the online shop. Out of all, only 13 companies have active online shops, of these, two via external services offered by companies specialized in the trading of wine products online. Among the main causes related to the lack of retails on line, there is certainly the complex logistics that derives from it, the complicated regulatory framework that surrounds the marketing of wine in different countries and, in all probability, also the excellent performance of the direct sales which discourages the implementation of such systems. The Langhe hills are characterized by a strong international presence of wine tourism, which guarantees the return of the customer in the area once or twice a year. Direct selling is a good source for companies with a dual relapse: firstly, it provides a greater margin of profit to the company that reduces logistics costs, commissions and all direct and indirect costs arising from the chains of traditional trade. Second, it allows a direct contact with the consumer, in order to establish a relationship which often is paid back by customer loyalty. Combined with direct sales on the spot is usually associated a visit and wine tasting. In fact, surfing the websites, we paid particular attention to the presence of information about the opportunity to visit the company, the procedures etc and we found 66 companies with a dedicated section. The modalities of participation vary from company to company, the only common feature is the requirement of a reservation. As we will see later, however, in general, almost all the companies are more than happy to host customers because very often such activities lead to the purchase of the products. We also found that more than 47 of these companies are able to offer accommodation for an overnight stay and in many cases also for lunches and dinners at the winery or in an adjacent room. In fact, many companies are developing a tourist offer, often using portions of farmsteads trying to create a welcoming environment, but, above all, by giving the chance to the customer a way to mix a simple visit with a real own experience within the company. In many cases, they offer meals made from local products paired with wines, walks and visits between the rows of vines to discover breathtaking landscapes. These activities allow the company to increase customer loyalty by offering unique and unforgettable experiences much appreciated especially by international customers.

Another point of analysis is the assessment of the presence of blogs. Blogs are a good way to share information; they are managed by a person, usually the owner, which explains some characteristics of the products, information on fairs and exhibition, and other 
important information. Blogs among companies have not spread much yet: only 4 blogs were found on the websites. In at least two cases, there is a blog run by the owner with the purpose of bringing customers to his products and company. Most of the submitted news, gossip or information are related to the consumption of products such as recipes and recommended combinations, prizes awarded and moments of life and emotions experienced during everyday business management. More widespread are video presentations of products and video reviews. This way, you can offer a presentation of the main characteristics of the product, highlighting the strengths and driving the consumer towards a proper interpretation of the product.

May we analyze the presence of a direct integration with social networks? The two most important are surely Facebook, which is currently the most used social network worldwide, and Twitter. There are also specialized portals, based on photo sharing, etc. From the standpoint of marketing, Facebook appears to be an excellent "weapon" for companies, regardless of their size. By creating fanpages on Facebook, companies create micro websites constantly updated, where users can post comments, links and images, look for information, or simply keep themselves up to date and view other users' experiences. The ubiquity of Facebook is huge: if we assume the page of a company with 2,000 fans, which have on average 300 friends, each new note in the web page can instantly reach approximately 600,000 people. Most companies have a Facebook page that appears to be the most used social network among the companies. Despite this, only 67 have a direct reference to a Facebook page. Generally this connection enables companies to share news and information quicker and in a simpler way than the website and certainly with a greater spread. The difference between a user profile and a fanpage from the point of view of functionality and the visibility on the network is huge. Within the sample, not all the companies can be found on social networks with their own fanpage easy to be accessed by users. About 12 companies are in fact recorded with a typical private profile, so that it is necessary to list them as friends in order to receive $100 \%$ of the different features. This factor is very limiting in terms of promotion. The Facebook pages are frequently updated with news, information, etc. The number of "likes" depends on the size of the company and it can vary from 100 up to 4000 people.

Twitter can be placed in the category of the microblogging. Used all around the world it is second only to Facebook. Twitter is based on the concept of followers or users who choose to follow other users through tweets, which allow you to stay updated on all their activities. Due to its simplicity, Twitter has experienced rapid expansion and today is used by Cuneo's wine makers. We count about 35 direct links to twitter profiles. There are 31 instead that are linked to other social networks like Instagram, Linkedin, Pinterest etc.

The next phase of the study was contacting each company through the email address posted on the website: this way it was possible to evaluate the attention devoted to such forms of contact and to measure response-time.
A total of 221 emails were sent, all with the same requests, with the pretention to be two clients who discovered the company and products through the website, we required the following information:

- Does the company carry mail orders?

- Are there are retailers / restaurants where you can buy / consume the wines in Rome?

- Is it possible to visit the winery and taste your products?

- What is the cost?

The response rate observed, which counted 145 responses to our requests $(65.6 \%)$, can be judged good enough. The other companies, even though they are on the network and have an email address, have not provided any answers. With further analysis we can say that the response rate as well, as the response times are directly related to the size of the company. In more than two cases, in fact, large production companies in the region have not provided any answers. All the companies contacted are available to be visited. Many offer a visit for free, while others set a token of participation which is always between 8 and 15 euro per person which is then deleted in case of purchase. Fewer are those which offer the visit for free with no obligation to purchase. Particular answer was given by a producer who said that the fixed cost of the visit varies according to the cost of the bottles that you choose to taste. As for Rome, the presence is quite good; there are some difficulties in finding the products of small businesses that often do not have capacities and experiences to be available throughout the country. Many companies, despite not having structured a shop on-line, say they are willing to make mail order sales, largely without establishing a minimum order. To affect the final cost, there are those due to the weight of the bottles especially for small quantities orders. Often, when purchasing by email is possible, an attachment with a price list was found in the same email. Let's look at the response times. $44.3 \%$ of the sample of 98 companies, has provided a response on the same day in which it was sent the e-mail message, $12.2 \%$ of 27 companies have provided information on the following day; $4.1 \%$ of 9 companies within two days and the remaining 5\% equal to 11 companies at a time over the two days. The responses given by family businesses often come directly from the owners, who give greater attention to the words used and the friendliness with which they are written.

\section{Conclusions}

The presence of companies on the net now appears to be widespread and allows everyone to reach users in all areas of the world. Not always the structure of the sites appears to be updated both from the content and the technological point of view. Some websites are now obsolete with outdated graphics. The use of new forms of marketing arrangements such as the exploitation of social networking and videos are not well-developed among the companies but will certainly be the most commonly used in the next years. The high response rate, completeness 
of the information provided and the rapidly with which they have reached maturity, make us realize that most of the companies positively judge the importance of contacts through its website. As the importance of the wine sector at the international level is high, companies in this area should give more attention to it. A similar study was carried out in the United States in 2009 questioning over 208 companies throughout the United States with particular attention to the state of California, very suited for viticulture, which represents $45 \%$ of the sample. If we try to compare the data we can see that the E-Commerce is much more developed in the United States with respect to the Langhe, where $61 \%$ of the companies has its own sales platform on-line. Despite this greater use of electronic commerce, the American companies have shown a lower response rate of $59 \%$ compared to $65.6 \%$ in the Langhe. Also in response time companies from Langhe region are quicker. In the United States at the time of the study, only
$14 \%$ answered the same day, $19 \%$ the next day, $13 \%$ within 2 days. The remaining $10 \%$ took more than two days to reply, while in Langhe only $5 \%$ of the times it took more than two days.

Of course, in 5 years, things could have changed so it would be interesting to make a comparison with the most current data. In the future, it would be good to extend the study to other wine areas in order to characterize the different areas and assess any discrepancies.

\section{References}

[1] L. Tacht, J. Wine Res, 20/2, 143-157 (2009)

[2] A.A.VV. , Slow Food Editore, Slow wine guida 2014 (2014)

[3] AA.VV., Gambero Rosso,Vini d'Italia 2014 (2014)

[4] AA.VV., Le guide de l'Espresso, I Vini d'Italia 2014, 28 (2014) 